\title{
The XENON Dark Matter programme: from XENON100 to XENON1T
}

\author{
MATTEO ALFONSI* for the XENON Collaboration \\ Nikhef, Science Park 105, Amsterdam, Netherlands \\ E-mail: malfonsi@nikhef.n]
}

The XENON Collaboration is presently operating the XENON100 dual-phase time projection chamber (TPC) at the LNGS underground laboratory in Italy to search for Weakly Interacting Massive Particles (WIMPs). The detector contains $161 \mathrm{~kg}$ of liquid xenon of which $62 \mathrm{~kg}$ is the WIMP target mass and the rest is used for shielding and active veto. The TPC capability of three dimensional vertex reconstruction and the combination of scintillation and ionisation signals allow for volume fiducialization and discrimination between electronic and nuclear recoils. In 2011, results based on 100.9 live days set the strongest limit on WIMP interactions at that time. Since then, new results were released in July 2012, with more than one calendar year of data, a lower krypton-related background, a lower trigger threshold and a longer electron lifetime. Based on a blind analysis of 224.6 live days $\times 34 \mathrm{~kg}$ exposure, no evidence of dark matter interaction was observed. The new results supersede the previous limit, excluding at $90 \%$ confidence level spin-independent WIMP-nucleon cross sections as low as $2 \times 10^{-45} \mathrm{~cm}^{2}$ at $55 \mathrm{GeV} / \mathrm{c}^{2}$ WIMP mass. The next phase of the programme will be accomplished with the XENON1T detector in construction at LNGS. The new detector will employ 2.4 tons of liquid xenon and will be placed in a water tank for further background reduction. The goal of XENON1T is to achieve a spin-independent cross section sensitivity of $\sim 2 \times 10^{-47} \mathrm{~cm}^{2}$ at a WIMP mass of $50 \mathrm{GeV} / \mathrm{c}^{2}$. I report on results from the operating XENON100 detector and describe the upcoming XENON1T experiment.

VIII International Workshop on the Dark Side of the Universe

June 10-15, 2012

Búzios, Rio de Janeiro, Brasil

\footnotetext{
* Speaker.
} 


\section{Introduction}

A long standing question of modern physics is the nature of Dark Matter (DM). It is still unknown, despite much astrophysical evidence for the existence of Dark Matter in the Universe. A very compelling hypothesis proposes that DM consists of subatomic Weakly Interacting Massive Particles (WIMPs) [1]. These would be particles with a mass between $1 \mathrm{GeV} / \mathrm{c}^{2}$ and $1 \mathrm{TeV} / \mathrm{c}^{2}$, only affected by gravity and the weak interaction. They would be long-lived and have been copiously produced in the early Universe. Several extensions of the Standard Model such as Supersymmetric Models might accommodate them.

WIMPs can occasionally interact elastically with nuclei: they could therefore be detected by observing the small recoils of such nuclei in a target material. These interactions would be exceedingly rare. Hence, several deep underground experiments with low radioactive background and high sensitivity to small energy deposits are competing world-wide with a variety of experimental techniques [2].

The XENON Collaboration leads a phased approach to WIMP direct detection employing ultra-pure liquid xenon as both target and detection medium. Liquid xenon (LXe) has ideal properties as a dark matter target [3]. The high mass of the xenon nucleus is favourable for scalar interactions and the presence of two isotopes with unpaired neutrons $\left({ }^{129} \mathrm{Xe}\right.$ : spin-1/2, $26.4 \%$ and ${ }^{131} \mathrm{Xe}$ : spin-3/2, 21.2\%) ensures sensitivity to axial WIMP-nuclei couplings. The high density $\left(3 \mathrm{~g} / \mathrm{cm}^{3}\right)$ and high atomic number $(\mathrm{Z}=54, \mathrm{~A}=131.3)$ allow to build self-shielding, compact dark matter detectors. As a detection medium, it has high scintillation and ionisation yields because of its low ionisation potential.

The XENON detectors, located at the Laboratori Nazionali del Gran Sasso (LNGS) in Italy, are dual-phase (liquid and vapour xenon) Time Projection Chambers (TPC) of increasing target mass and reduced background. XENON10 detector 㺻, employing a $\sim 10 \mathrm{~kg}$ target, excluded previously unexplored parameter space, setting $90 \%$ C.L. upper limits of $4.5 \times 10^{-44} \mathrm{~cm}^{2}$ and $5 \times 10^{-39} \mathrm{~cm}^{2}$ on the WIMP-nucleon spin-independent and spin-dependent cross-sections, respectively, at a WIMP mass of $30 \mathrm{GeV} / \mathrm{c}^{2}$ [5, 6]. The XENON100 detector, the current phase of the programme, is taking data at LNGS since 2008 [7]. The detector and the last science run are described in Sec. 国. At the same time, the construction of XENON1T in Hall B at LNGS is approved and will start in 2013. Sec. 3 gives details of its design.

\section{The XENON100 experiment}

The XENON100 detector, described in detail in [8], is a cylindrical $(30.6 \mathrm{~cm}$ diameter, $30.5 \mathrm{~cm}$ height) dual-phase TPC enclosing a LXe target mass of $62 \mathrm{~kg}$. Its walls are made of 24 interlocking polytetrafluorethylen (PTFE) panels, which work as insulators and are good reflectors for the xenon scintillation light. The electrical fields are created with thin metal meshes that were etched with a hexagonal pattern from stainless steel foils and spot-welded onto low-radioactivity, stainless steel rings. An additional $99 \mathrm{~kg}$ of the same high-purity LXe, optically separated from the target volume, is instrumented as an active scintillator veto. The TPC and the veto are mounted in a double-walled stainless-steel cryostat, enclosed by a passive shield made from oxygen-free highconductivity copper, polyethylene, lead, and water/polyethylene. The shield is permanently purged 
with boil-off nitrogen gas in order to suppress radon backgrounds. The LXe is kept at the operating temperature of about $-91{ }^{\circ} \mathrm{C}$ by a pulse tube refrigerator (PTR) mounted outside the shield. Fig. 1 shows the drawing of the detector and a schematic view of its passive shield.
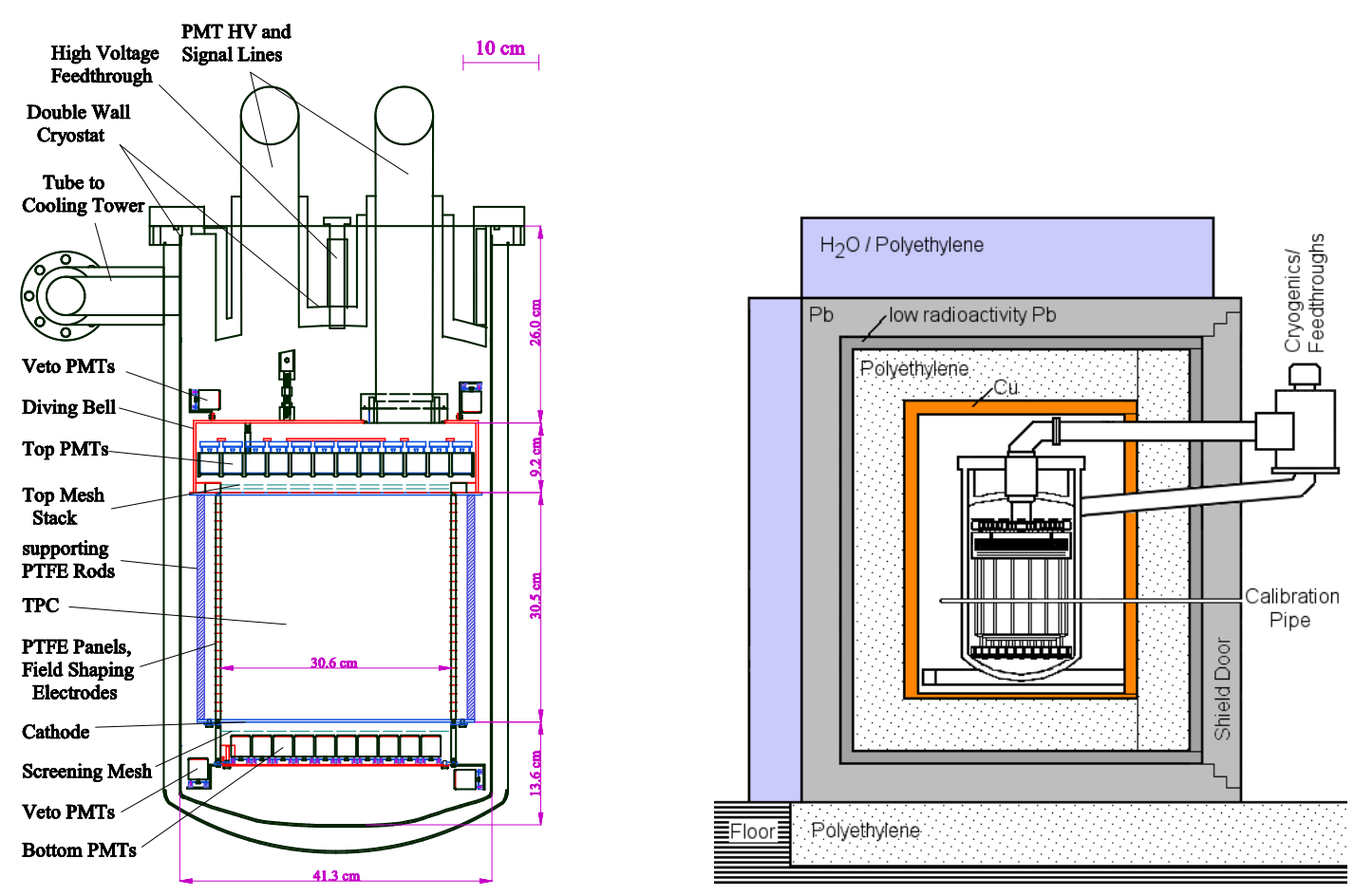

Figure 1: (Left): Drawing of the XENON100 detector. The time projection chamber contains $62 \mathrm{~kg}$ of liquid xenon and is surrounded by an active liquid xenon veto of $99 \mathrm{~kg}$. (Right): Schematic view of XENON100 in its passive shield made of copper, polyethylene, lead and water [8].

An energy deposition in the liquid xenon produces both ionisation electrons and scintillation photons. The ratio of the two signals is different for nuclear recoils (NR, created by WIMP or neutron interactions) and electronic recoils (ER, produced by beta and gamma rays), providing the basis of one of the major background discrimination techniques. The electrons, moved from the interaction point by a drift field of $530 \mathrm{~V} / \mathrm{cm}$, are extracted from the liquid and accelerated in the gas by a $\sim 12 \mathrm{kV} / \mathrm{cm}$ field, producing proportional scintillation light. The amplified charge signal (S2) and the direct scintillation signal (S1) are both detected by two arrays of 1"-square Hamamatsu R8520-AL photomultipliers (PMTs), selected for low radioactivity and high quantum efficiency. One array is immersed in the LXe below the cathode of the TPC for optimal light collection, and one is placed in the xenon gas above the amplification gap. The $z$-position of a particle interaction in the TPC is reconstructed, with a precision of $0.3 \mathrm{~mm}(1 \sigma)$, from the time difference between the $\mathrm{S} 1$ and $\mathrm{S} 2$ signals and the known electron drift velocity $(1.73 \mathrm{~mm} / \mu \mathrm{s})$. The localised distribution of the S2 signal over the top PMTs is used to obtain the $(x, y)$-coordinates using a neural network algorithm with an uncertainty $<3 \mathrm{~mm}(1 \sigma)$. The spatial reconstruction also allows for the rejection of multiple-scatter events, such as from neutrons, since WIMPs are expected to interact only once. Double-scatter events can be separated when their vertices differ by $\Delta z>3 \mathrm{~mm}$.

PMT gains are determined by stimulating single photoelectron emission from the photocath- 
ode using light pulses from a blue LED $(\lambda=470 \mathrm{~nm})$, driven by a pulse generator. The average gain was stable at $2 \times 10^{6}$ within $\pm 1 \%$ during science runs.

Calibration sources can be inserted in the XENON100 shield through a copper tube which is wound around the cryostat (see Fig. 1, right). While the vertical source position is restricted to the TPC centre, it can be placed at all azimuthal angles. The electronic recoil band in $\log _{10}(\mathrm{~S} 2 / \mathrm{S} 1)$ vs energy space defines the region of background events from $\beta$ - and $\gamma$-particles. It is measured using the low energy Compton tail of high-energy $\gamma$-sources such as ${ }^{60} \mathrm{Co}$ and ${ }^{232} \mathrm{Th}$. The response to single scatter nuclear recoils, which is the expected signature of a WIMP, is measured with an ${ }^{241} \mathrm{AmBe}(\alpha, \mathrm{n})$-source, shielded by $10 \mathrm{~cm}$ of lead in order to suppress the contribution from its high energy gamma rays $(4.4 \mathrm{MeV})$. Besides the definition of the nuclear recoil band and a benchmark WIMP search region, this calibration provides additional gamma lines from inelastic scattering as well as from xenon or fluorine activation at $40 \mathrm{keV}\left({ }^{129} \mathrm{Xe}\right), 80 \mathrm{keV}\left({ }^{131} \mathrm{Xe}\right), 110 \mathrm{keV}$ $\left({ }^{19} \mathrm{~F}\right.$ in PTFE), $164 \mathrm{keV}\left({ }^{131 \mathrm{~m}} \mathrm{Xe}\right), 197 \mathrm{keV}\left({ }^{19} \mathrm{~F}\right)$ and $236 \mathrm{keV}\left({ }^{129 \mathrm{~m}} \mathrm{Xe}\right)$.

\subsection{The last XENON100 science run}

The last science run collected a dark matter dataset of 224.6 live days between February 2011 and March 2012. The PTR was in continuous operation for a total of $\sim 20$ months. This is the first demonstration, to our knowledge, of a LXe detector operated over such a long period of time.

In 2011 the XENON Collaboration published results for spin-independent [9] and inelastic [10] WIMP-nucleon cross-sections based on the 100.9 live days science run of 2010. The data analysis is described in [11]. The analysis procedure for the recently released run is similar. However the run is characterised by improved experimental conditions.

We have obtained a substantial reduction of the intrinsic background from ${ }^{85} \mathrm{Kr}$ by cryogenic

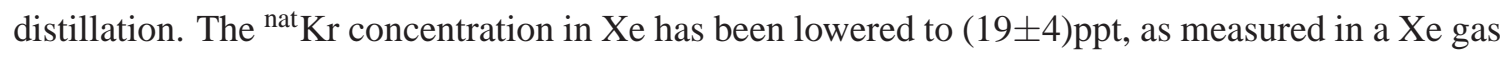
sample from the detector using ultra-sensitive rare gas mass spectrometry combined with a $\mathrm{Kr} / \mathrm{Xe}$ separation technique. This is consistent with the (18 \pm 8$)$ ppt derived from counting the number of delayed $\beta-\gamma$ coincidences associated with the ${ }^{85} \mathrm{Kr}$ beta decay, assuming a ${ }^{85} \mathrm{Kr} /{ }^{\text {nat }} \mathrm{Kr}$ ratio of $2 \times 10^{-11}$ [12].

The data have been acquired under improved electronic noise conditions and with a modified hardware trigger to allow for a reduced S2 trigger threshold for $>99 \%$ of S2 signals above 150 photo-electrons (PE) to be recorded. This leads to virtually no loss of events in the energy region of interest.

The larger amount of calibration data, collected on regular a basis throughout the DM data taking period, has allowed for the improvement of the detector response corrections at the percentlevel accuracy. Indeed the non-uniform light collection by the two PMT arrays and the attenuation of the ionisation signal by residual impurities over the maximum drift gap of $30 \mathrm{~cm}$ lead to a position-dependent $\mathrm{S} 1$ and $\mathrm{S} 2$ signal response. The signals are corrected using maps derived from calibration data. The $\mathrm{S} 1$ light yield is 3 -dimensionally corrected in cylindrical coordinates $(r ; \theta ; z)$ in order to optimise the response very close to the PMTs. Consistent correction maps are obtained with the $662 \mathrm{keV}$ photopeak of the ${ }^{137} \mathrm{Cs}$ source decay, or with the neutron-activated Xe lines at $40 \mathrm{keV}$ and $164 \mathrm{keV}$. The electron lifetime $\tau_{e}$, used to describe the ionisation loss by impurities in LXe, has been measured once per week with a ${ }^{137} \mathrm{Cs}$ source. The value has increased from $374 \mu \mathrm{s}$ to $611 \mu \mathrm{s}$, with the average being $\tau_{e}=514 \mu \mathrm{s}$. The measured drift time $t_{d}$ is used to correct 
the $\mathrm{S} 2$ signal size for these losses, and an additional correction in $(x ; y)$ accounts for variations due to photon collection efficiency and small inhomogeneities in the mesh electrodes. Finally, a considerable effort within the analysis has been devoted on the rejection cuts against electronic noise.

As in the previous run, it was decided a priori to use a Profile Likelihood (PL) statistical inference method to set WIMP limits [13]. Signal (NR) and background (ER) events can be distinguished by their different S2/S1 ratio, where only the S2 signal detected by the bottom PMTs, $\mathrm{S} 2_{\mathrm{b}}$, is used since it requires smaller corrections [8]. The mean of the $\log _{10}\left(\mathrm{~S} 2_{\mathrm{b}} / \mathrm{S} 1\right)$ band from ER calibration data is subtracted in order to remove the energy-dependence of this discrimination parameter.

The WIMP search is restricted to the energy window 3-30 PE, which in nuclear recoil equivalent energy corresponds to $6.6-43.3 \mathrm{keV}_{\mathrm{nr}}$. The energy scale for NRs is derived from the S1 signal using the independently measured relative scintillation efficiency $\mathscr{L}_{\text {eff }}$ via the relation $E_{\text {nr }}=\left(S 1 / L_{\mathrm{y}}\right)\left(1 / \mathscr{L}_{\text {eff }}\right)\left(S_{\text {ee }} / S_{\text {nr }}\right)$ (see [14] and references therein). The $\mathscr{L}_{\text {eff }}$ parametrisation of [9] is used. The fiducial volume used in this analysis contains $34 \mathrm{~kg}$ of LXe. The volume was determined by maximising the sensitivity of this run given the accessible ER background above the blinding cut.

The blinding procedure consisted of masking the dark matter data from 2-100 PE in S1 by keeping only the upper $90 \%$ of the ER band, hiding more than $90 \%$ of the signal region. In a predefined benchmark WIMP search region with shorter energy range $6.6-30.5 \mathrm{keV}_{\mathrm{nr}}$ the expected number of $\mathrm{BG}$ events were $1.0 \pm 0.2$. After the unblinding, two events have been observed, as shown in Fig. 2. In this figure, we can also observe the negligible impact of the S2 > $150 \mathrm{PE}$ threshold cut which is indicated by the dashed-dotted blue curve. The signal region is also restricted by a lower border running along the $97 \%$ NR quantile (dashed blue curve).

The PL analysis yields a p-value of $\geq 5 \%$ for all WIMP masses for the background-only hypothesis indicating that there is no excess due to a dark matter signal. Under standard assumptions of the Dark Matter halo [15], 90\% confidence level exclusion limit for the spin-independent WIMPnucleon cross section $\sigma_{\mathrm{SI}}$ is calculated. The expected sensitivity of this dataset in the absence of any signal is shown by the green $(1 \sigma)$ and yellow $(2 \sigma)$ bands in Fig. 3 . The new limit is represented by the thick blue line. It excludes a large fraction of previously unexplored parameter space, including regions preferred by scans of the constrained supersymmetric parameter space [16]. The new XENON100 data provide the most stringent limit for $m_{\mathrm{WIMP}}>8 \mathrm{GeV} / c^{2}$ with a minimum of $\sigma_{\mathrm{SI}}=2.0 \times 10^{-45} \mathrm{~cm}^{2}$ at $m_{\mathrm{WIMP}}=55 \mathrm{GeV} / \mathrm{c} 2$. The new XENON100 result continues to challenge the interpretation of the DAMA [17], CoGeNT [18], and CRESST-II [19] results as being due to scalar WIMP-nucleon interactions.

\section{The XENON1T detector}

In parallel to the successful operations of XENON100, the Collaboration is in the process of finalising the design and starting construction of the next generation detector, XENON1T, with a fiducial mass of about 1 ton. The construction in Hall B at LNGS will start in 2013. It essentially scales up the existing XENON100 detector by about a factor of 20 and reduces the background by three orders of magnitude. The detector is a cylindrical dual-phase TPC with $1 \mathrm{~m}$ diameter 


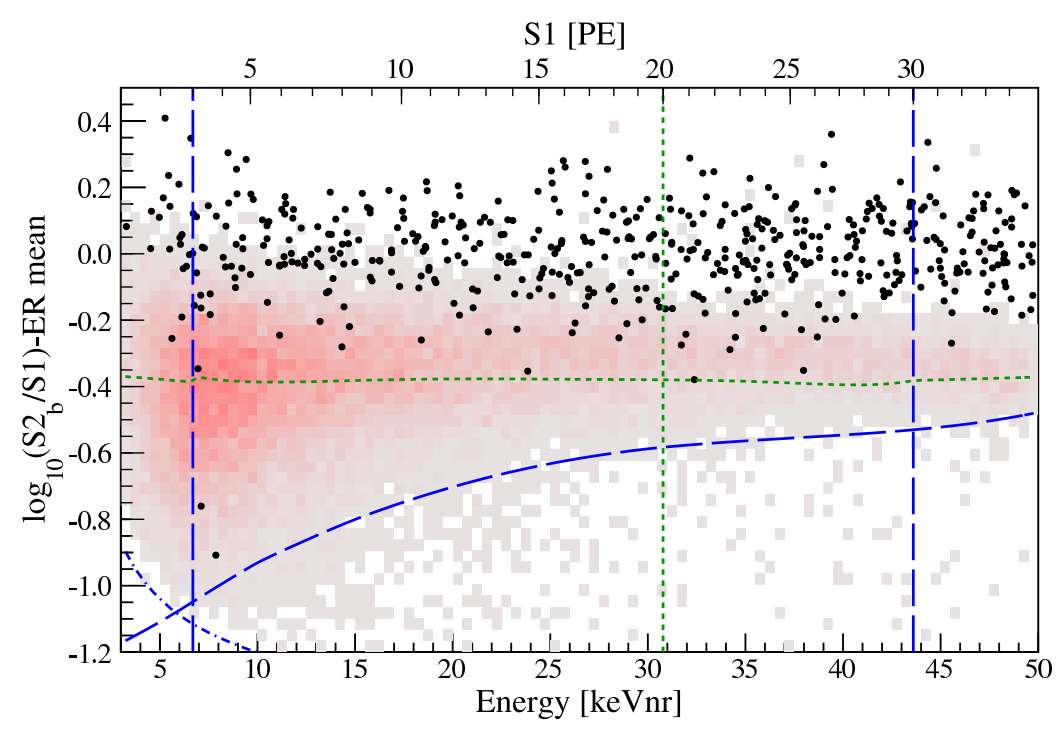

Figure 2: Event distribution in the discrimination parameter space $\log _{10}\left(\mathrm{~S} 2_{\mathrm{b}} / \mathrm{S} 1\right)$, flattened by subtracting the distribution's mean, as observed after unblinding using all analysis cuts and a $34 \mathrm{~kg}$ fiducial volume (black points). A lower analysis threshold of $6.6 \mathrm{keV}_{\mathrm{nr}}$ (NR equivalent energy scale) is employed. The PL analysis uses an upper energy threshold of $43.3 \mathrm{keV}_{\mathrm{nr}}(3-30 \mathrm{PE})$ and the benchmark WIMP search region is limited to $30.5 \mathrm{keV}_{\mathrm{nr}}(3-20 \mathrm{PE})$. The negligible impact of the $\mathrm{S} 2>150 \mathrm{PE}$ threshold cut is indicated by the dashed-dotted blue line and the signal region is restricted by a lower border running along the $97 \%$ NR quantile. An additional hard S2 $/ \mathrm{S} 1$ discrimination cut at $99.75 \%$ ER rejection defines the benchmark WIMP search region from above (dotted green) but is only used to cross check the PL inference. The histogram in red indicates the NR band from the neutron calibration. Two events fall into the benchmark region where $(1.0 \pm 0.2)$ are expected from background.

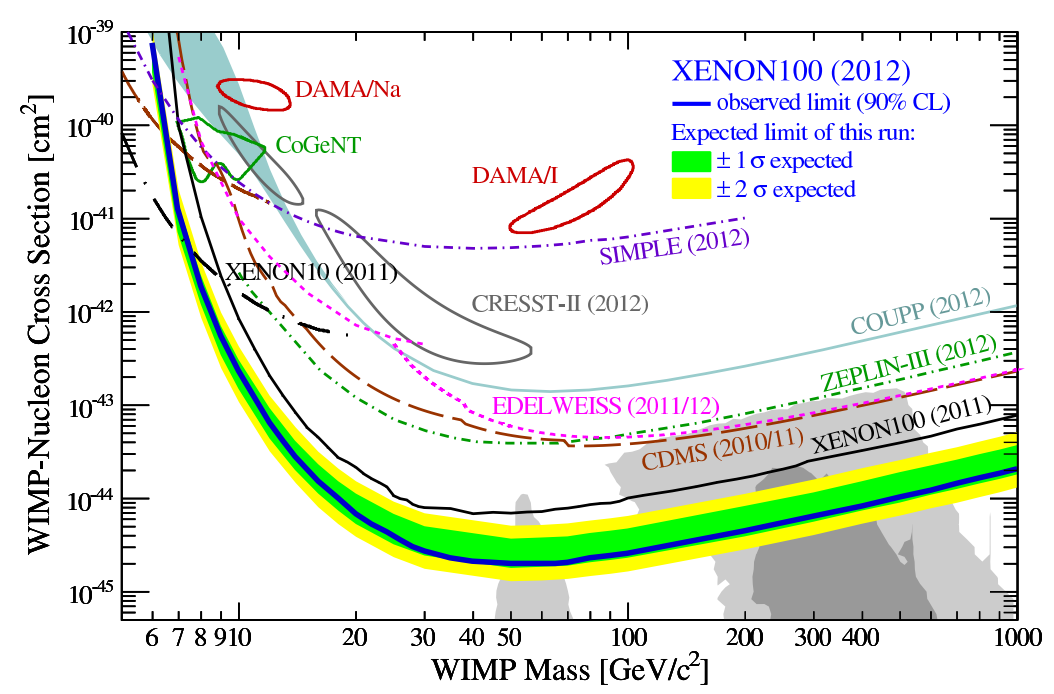

Figure 3: New result on spin-independent WIMP-nucleon scattering from XENON100: the expected sensitivity of this run is shown by the green/yellow band $(1 \sigma / 2 \sigma)$ and the resulting exclusion limit (90\% CL) in blue. For comparison, other experimental results [17, 18, 19] are also shown, together with the regions $(1 \sigma / 2 \sigma)$ preferred by supersymmetric (CMSSM) models [16]. 
and $1 \mathrm{~m}$ drift length, containing $\sim 2.4$ ton of pure LXe and designed to operate with a $1 \mathrm{kV} / \mathrm{cm}$ drift field. It is instrumented with approximately 250 3"-diameter PMTs (Hamamatsu R11410 series). The cryostat is suspended within a $9.6 \mathrm{~m}$ diameter water tank. The water acts as shield and active Cherenkov muon veto with $4 \pi$ coverage for the XENON1T detector. It is very effective in reducing the neutron and $\gamma$-ray background from the underground rock cavern or from cosmic muon to negligible levels.

The muon veto, an extensive material screening campaign, the reduction of the intrinsic background coming from $\mathrm{Kr}$ and $\mathrm{Rn}$ contamination and the self-shielding properties of liquid xenon allow to achieve the goal of less than 1 background event per 2 ton-year exposure. At the same time, with 2 years live-time and $\sim 1$ ton fiducial mass, XENON1T could detect on the order of 100 dark matter events, assuming a spin independent WIMP-nucleon cross section $\sigma_{\mathrm{SI}}$ of $\sim 10^{-45} \mathrm{~cm}^{2}$ and for a WIMP mass of $100 \mathrm{GeV} / \mathrm{c}^{2}$. In the absence of a positive signal, the experiment aims to exclude cross-sections above $\sigma_{\mathrm{SI}} \sim 2 \times 10^{-47} \mathrm{~cm}^{2}$ at $90 \% \mathrm{CL}$ for $50 \mathrm{GeV} / \mathrm{c}^{2}$ WIMPs.

\section{References}

[1] G. Bertone, D. Hooper, J. Silk, Particle Dark Matter: Evidence, Candidates and Constraints, Phys. Rept. 405 (2005) 279-390 [hep-ph/ 0404175 ]

[2] R. W. Schnee, Introduction to dark matter experiments, In Physics of the Large and Small: Proceedings of the 2009 Theoretical Advanced Study Institute in Elementary Particle Physics, World Scientific, Singapore (2011) 629-681 [arXiv:1101.5205] .

[3] E. Aprile, T. Doke, Liquid xenon detectors for particle physics and astrophysics, Rev. Mod. Phys. 82 (2010) 2053.

[4] E. Aprile et al. (XENON10 Collaboration), Design and performance of the XENON10 dark matter experiment Astroparticle Physics 34 (2011) 679-698.

[5] J. Angle et al. (XENON Collaboration), First Results from the XENON10 Dark Matter Experiment at the Gran Sasso National Laboratory, Phys. Rev. Lett. 100 (2008) 021303.

[6] J. Angle, E. Aprile, F. Arneodo, L. Baudis, A. Bernstein, A. Bolozdynya, L. C. C. Coelho, C. E. Dahl et al., Limits on Spin-Dependent WIMP-Nucleon Cross Sections from the XENON10 Experiment, Phys. Rev. Lett. 101 (2008) 091301.

[7] E. Aprile et al. (XENON100 Collaboration), First Dark Matter Results from the XENON100 Experiment, Phys. Rev. Lett. 105 (2010) 131302.

[8] E. Aprile et al. (XENON100 Collaboration), The XENON100 Dark Matter Experiment, Astroparticle Physics 35 (2012) 573-590.

[9] E. Aprile et al. (XENON100 Collaboration), Dark Matter Results from 100 Live Days of XENON100 Data, Phys. Rev. Lett. 107 (2011) 131302.

[10] E. Aprile et al. (XENON100 Collaboration), Implications on Inelastic Dark Matter from 100 Live Days of XENON100 Data, Phys. Rev. D 84 (2011) 061101.

[11] E. Aprile et al. (XENON100 Collaboration), Analysis of the XENON100 Dark Matter Search Data, arXiv: 1207.5988 (2012), submitted to Astroparticle Physics.

[12] X. Du et al., An atom trap system for practical ${ }^{81} \mathrm{Kr}$ dating, Rev. Sci. Instr. 75 (2004) 3224. 
[13] E. Aprile et al. (XENON100 Collaboration), Likelihood Approach to the First Dark Matter Results from XENON100, Phys. Rev. D 84 (2011) 052003.

[14] G. Plante et al., New Measurement of the Scintillation Efficiency of Low-Energy Nuclear Recoils in Liquid Xenon, Phys. Rev. C 84 (2011) 045805.

[15] E. Aprile et al. (XENON100 Collaboration), Dark Matter Results from 225 Live Days of XENON100 Data, Phys. Rev. Lett. 109 (2012) 181301 [arXiv:1207.5988]

[16] Combined region using C. Strege et al., JCAP 1203, 030 (2012); A. Fowlie et al. (2012), arXiv:1206.0264; O. Buchmueller et al. (2011), arXiv:1112.3564.

[17] C. Savage et al., Compatibility of DAMA/LIBRA dark matter detection with other searches, JCAP 0904, 010 (2009).

[18] C. E. Aalseth et al. (CoGeNT), Results from a Search for Light-Mass Dark Matter with a p-Type Point Contact Germanium Detector, Phys. Rev. Lett. 106 (2011) 131301.

[19] G. Angloher et al. (CRESST-II), Results from $730 \mathrm{~kg}$ days of the CRESST-II Dark Matter search, Eur. Phys. J. C 72 (2012) 1971. 